\title{
MPE-BASED DISCRIMINATIVE LINEAR TRANSFORM FOR SPEAKER ADAPTATION
}

\author{
L. Wang and P.C. Woodland \\ Machine Intelligence Laboratory, \\ Cambridge University Engineering Department, \\ Trumpington Street, Cambridge, CB2 1PZ, UK. \\ Email: $\{1 w 256, p c w\} @ e n g . c a m . a c . u k$
}

\begin{abstract}
In this paper, we present a discriminative method for speaker adaptation, where the minimum phone error (MPE) criterion is used to estimate the discriminative linear transform (DLT), including mean and diagonal variance transforms. The I-smoothing technique is essential to improve the generalization of DLTs. Experiments on supervised adaptation for non-native speakers on the North American Business (NAB) Spoke 3 task show that MPEbased DLT outperforms both MLLR and previously proposed discriminative method for transform estimation. Preliminary experiments on unsupervised DLT estimation are plotted on conversational telephone speech transcription.
\end{abstract}

\section{INTRODUCTION}

Speaker adaptation is crucial to produce a speaker-specific system based upon the speaker-independent HMM sets, given a small amount of adaptation utterances. For a variety of adaptation tasks, maximum likelihood linear regression (MLLR) for model-space transformation is an effective and efficient approach. MLLR can use a so-called regression-class tree to adjust the number of generated transforms, according to the amount of adaptation data available. Using the maximum likelihood (ML) criterion to estimate the transform parameters, MLLR can be used to estimate mean, diagonal variance, or full variance transforms, for the HMM parameters. MLLR can be operated in either supervised or unsupervised mode.

Since discriminative training criteria [5] such as maximum mutual information (MMI) and minimum phone error (MPE) $[6,7]$ have been successfully used to train HMM-based acoustic models, it is then expected that the same discriminative criteria can benefit the estimation of the linear transforms for both adaptive training [10] and adaptation. In related work [3], the H-criterion was used to estimate discriminative linear transform (DLT), where the objective function was designed to be an interpolation of ML and MMI. The conditional maximum likelihood linear regression [4], another derivation of the MMI criterion, was also proposed for transform generation. Recently, using the minimum classification error (MCE) to estimate the diagonal variance transform has also been explored and reported in [9]. We also investigated the use of the MPE criterion for constrained DLT, which was then applied to the features for discriminative speaker adaptive training [10].

Experiments on conversational telephone speech (CTS) transcription for the direct estimation of HMM parameters have demon-

This work was supported by DARPA grant MDA972-02-1-0013. The paper does not necessarily reflect the position or the policy of the US Government and no official endorsement should be inferred. strated that MPE training reliably outperforms MMI training on test data, since error driven criteria such as MPE, focus on correctable errors in the training data rather than outliers which may reduce the effectiveness of MMI training. Therefore in this paper, we concentrate on using the MPE criterion for linear transform estimation, including mean and diagonal variance transform estimation, and investigate the use of MPE-based DLT for both supervised and unsupervised speaker adaptation. The derivation for MPE-DLT estimation relies on the use of weak-sense auxiliary functions. Furthermore it is necessary to smooth the discriminative statistics with those used for ML estimation. This I-smoothing [6] can also be used to improve the generalization of MPE-based DLT.

The effectiveness of MPE-based DLT estimation is first evaluated in the context of supervised adaptation to non-native speakers from a HMM set trained on native speakers and uses the North American Business News Spoke 3 task. We have then investigated the used of discriminative estimation for unsupervised adaptation, which by the nature of discriminative training techniques is a difficult problem.

The rest of this paper is organized as below. In Section 2, we describe the MPE criterion for DLT estimation, including the use of a weak-sense auxiliary function for the optimization and statistics smoothing technique. The supervised adaptation results on WSJ task are presented in Section 3. For unsupervised style, the experiments are carried out on CTS transcription. Then some issues concerning MPE-based DLT for unsupervised adaptation are discussed in the last section.

\section{THE MPE CRITERION FOR DISCRIMINATIVE LINEAR TRANSFORM}

Recently proposed for continuous speech recognition, the MPE criterion was designed to evaluate the phone accuracy in the word context. The MPE objective function was defined in [6,7],

$$
\mathcal{F}_{M P E}(\lambda)=\sum_{r=1}^{R} \frac{\sum_{\hat{w}} P_{\lambda}\left(\mathcal{O}_{r} \mid \mathcal{M}^{\hat{w}}\right)^{\kappa} P(\hat{w}) \text { RawAccuracy }(\hat{w})}{\sum_{w} P_{\lambda}\left(\mathcal{O}_{r} \mid \mathcal{M}^{w}\right)^{\kappa} P(w)}
$$

where $\mathcal{M}^{w}$ is the composite model corresponding to the word sequence $w, P(w)$ is the probability of the word sequence $w$ and $\kappa$ is the acoustic scale. The RawAccuracy $(\hat{w})$ measures the number of phones correctly recognized in the sentence $\hat{w}$.

In the implementation of MPE training, lattices marked with time information at HMM level are used to represent the correct transcriptions and confusable hypotheses from recognition, as in 
MMI training. Using lattices can reduce the computational load for generating the statistics needed for parameter estimation [5].

\subsection{The optimization of objective function}

For the optimization of discriminative criteria, the weak-sense auxiliary function was proposed $[8,7]$, in contrast to the use of the standard strong-sense auxiliary function [7] for ML training. Given the objective function $\mathcal{F}(\lambda)$, the weak-sense auxiliary function is defined to satisfy the following condition:

$$
\left.\frac{\partial}{\partial \hat{\lambda}} \mathcal{Q}(\hat{\lambda}, \lambda)\right|_{\hat{\lambda}=\lambda}=\left.\frac{\partial}{\partial \hat{\lambda}} \mathcal{F}(\hat{\lambda})\right|_{\hat{\lambda}=\lambda}
$$

where $\lambda$ refers to the original parameter set and $\hat{\lambda}$ represents the newly estimated one. This equation implies that if there is a local maximum in the objective function, it must also be a local maximum of the auxiliary function. Although optimizing the weaksense auxiliary function doesn't guarantee an increase in the objective function, it can still offer the minimum condition for the optimization of $\mathcal{F}(\lambda)$. For discriminative training, the weak-sense auxiliary function provides a feasible approach to optimize the objective functions with negative terms.

Concerning the phone accuracy in the MPE objective function, the auxiliary function proposed in $[8,7]$ is then based on the log likelihood of phone arc to make the optimization tractable,

$$
\mathcal{G}_{M P E}(\lambda, \hat{\lambda})=\left.\sum_{r=1}^{R} \sum_{q=1}^{Q_{r}} \frac{\partial \mathcal{F}_{M P E}}{\partial \log p(q)}\right|_{(\lambda=\hat{\lambda})} \log p(q) .
$$

Here each sentence $r$ contains a set of phone $\operatorname{arcs} q=1, \ldots, Q_{r}$, and $p(q)$ represents the likelihood of arc $q$ calculated from the corresponding starting to ending times.

Eq. (2) can be separated into two parts in terms of the positive and negative values of $\left.\frac{\partial \mathcal{F}_{M P E}}{\partial \log p(q)}\right|_{(\lambda=\hat{\lambda})}$, which are analogous to the numerator and denominator terms in the MMI auxiliary function. More important, it is proven that the model parameter updating formulations have the similar forms as that in MMI training, provided that the numerator/denominator statistics have different definitions[7].

When using the MPE criterion to estimate linear transforms, a weak-sense auxiliary function is thus presented for the optimization. As standard MLLR [2], MPE-based DLT is applied to transform Gaussian means with a matrix $\mathbf{A}$ and a bias $\mathbf{b}$,

$$
\tilde{\mu}_{m}=\mathbf{A} \mu_{m}+\mathbf{b}=W \xi_{m},
$$

where $W=\left[\begin{array}{ll}\mathbf{b} & \mathbf{A}\end{array}\right], \xi_{m}=\left[\begin{array}{ll}1 & \mu_{m}{ }^{T}\end{array}\right]^{T}$. With the quantity defined for MPE training, $\gamma_{q}^{M P E}=\frac{1}{\kappa} \frac{\partial \mathcal{F}_{M P E}}{\partial \log p(q)}$, the auxiliary function consists of three individual parts, each of which has a Gaussian expression,

$$
\begin{aligned}
& \mathcal{G}_{M P E}(W, \hat{W}) \\
= & \sum_{r=1}^{R} \sum_{q=1}^{Q} \sum_{t=s_{q}}^{t=e_{q}} \gamma_{q m}(t) f\left(\gamma_{q}^{M P E}\right) \log \mathcal{N}\left(\mathbf{o}(t), \hat{W} \xi_{m}, \Sigma_{m}\right) \\
- & \sum_{r=1}^{R} \sum_{q=1}^{Q} \sum_{t=s_{q}}^{t=e_{q}} \gamma_{q m}(t) f\left(-\gamma_{q}^{M P E}\right) \log \mathcal{N}\left(\mathbf{o}(t), \hat{W} \xi_{m}, \Sigma_{m}\right) \\
+ & \mathcal{G}_{s m}(W, \hat{W})
\end{aligned}
$$

where $\gamma_{q m}(t)$ is the posterior probability over time $t$, at state $j$, mixture component $m$ on condition of arc $q$. The function $f\left(\gamma_{q}^{M P E}\right)$ defined as below determines that the arcs with positive $\gamma_{q}^{M P E}$ will be used to accumulate the numerator statistics, while those with negative values will be used to get denominator statistics.

$$
\left\{\begin{array}{c}
f\left(\gamma_{q}^{M P E}\right)=\max \left(0, \gamma_{q}^{M P E}\right) \\
f\left(-\gamma_{q}^{M P E}\right)=\max \left(0,-\gamma_{q}^{M P E}\right)
\end{array}\right.
$$

The smoothing function in Eq. (3) associates with the initial adapted model parameters to improve the stability of training,

$$
\begin{aligned}
& \mathcal{G}_{s m}(W, \hat{W})=\sum_{m} D_{m}\left[-\frac{1}{2}\left(\log \left|\hat{\Sigma}_{m}\right|\right.\right. \\
& \left.\left.+\left(W \xi_{m}-\hat{W} \xi_{m}\right)^{T} \hat{\Sigma}_{m}^{-1}\left(W \xi_{m}-\hat{W} \xi_{m}\right)+\Sigma_{m} \hat{\Sigma}_{m}^{-1}\right)\right]
\end{aligned}
$$

Obviously the differential of this smoothing function at $\hat{W}=W$ is zero, so that adding this function ensures that the whole auxiliary function still satisfies the condition for weak-sense definition. And $D_{m}$ is defined as the smoothing factor with a constant $E$,

$$
D_{m}=E \sum_{q=1}^{Q} \sum_{t=s_{q}}^{t=e_{q}} \gamma_{q m}(t) f\left(-\gamma_{q}^{M P E}\right) .
$$

\subsection{MPE-based discriminative linear transformation}

Calculating the partial differential of Eq. (3) with respect to each row of the linear transform $\hat{\mathbf{w}}^{(i)}$ yields a close solution:

$$
\begin{aligned}
\hat{\mathbf{w}}^{(i)} & =\mathbf{G}^{(i)^{-1}} \mathbf{k}^{(i)} \\
\mathbf{G}^{(i)} & =\sum_{m} \frac{1}{\sigma_{m(i)}^{2}}\left(\left(\gamma_{m}^{n u m}-\gamma_{m}^{d e n}\right)+D_{m}\right) \xi_{m}^{T} \xi_{m} \\
\mathbf{k}^{(i)} & =\sum_{m} \frac{1}{\sigma_{m(i)}^{2}}\left(\theta_{m}^{n u m}\left(\mathcal{O}_{(i)}\right)-\theta_{m}^{d e n}\left(\mathcal{O}_{(i)}\right)+D_{m} \tilde{\mu}_{m(i)}\right) \xi_{m}^{T}
\end{aligned}
$$

Here, the numerator/denominator statistics to estimate MPE-based DLT have the following forms,

$$
\begin{aligned}
\gamma_{m}^{n u m} & =\sum_{q=1}^{Q} \sum_{t=s_{q}}^{t=e_{q}} \gamma_{q m}(t) f\left(\gamma_{q}^{M P E}\right) \\
\theta_{m}^{n u m}(\mathcal{O}) & =\sum_{q=1}^{Q} \sum_{t=s_{q}}^{t=e_{q}} \gamma_{q m}(t) f\left(\gamma_{q}^{M P E}\right) \mathbf{o}(t) \\
\theta_{m}^{n u m}\left(\mathcal{O}^{2}\right) & =\sum_{q=1}^{Q} \sum_{t=s_{q}}^{t=e_{q}} \gamma_{q m}(t) f\left(\gamma_{q}^{M P E}\right) \mathbf{o}^{2}(t), \\
\gamma_{m}^{d e n} & =\sum_{q=1}^{Q} \sum_{t=s_{q}}^{t=e_{q}} \gamma_{q m}(t) f\left(-\gamma_{q}^{M P E}\right) \\
\theta_{m}^{d e n}(\mathcal{O}) & =\sum_{q=1}^{Q} \sum_{t=s_{q}}^{t=e_{q}} \gamma_{q m}(t) f\left(-\gamma_{q}^{M P E}\right) \mathbf{o}(t) \\
\theta_{m}^{d e n}\left(\mathcal{O}^{2}\right) & =\sum_{q=1}^{Q} \sum_{t=s_{q}}^{t=e_{q}} \gamma_{q m}(t) f\left(-\gamma_{q}^{M P E}\right) \mathbf{o}^{2}(t) .
\end{aligned}
$$


The smoothing factor $D_{m}=E \gamma_{m}^{d e n}$ is given as in [11], $E$ is a constant between 1 to 2 (selected by empirical results), and $\tilde{\mu}_{m}$ is the adapted mean vectors with the initial MLLR transform $W$.

Moreover, we can also derive the diagonal variance transform $\hat{\mathbf{H}}$ under MPE criterion after applying the newly estimated mean transforms $\hat{W}$. As ML-based diagonal variance transform, the MPE-based diagonal variance transform is used to transform the diagonal variance,

$$
\hat{\Sigma}_{m}=\mathbf{H}^{T} \Sigma_{m} \mathbf{H}, \quad \hat{\Sigma}_{m}^{-1}=\mathbf{L}_{m}^{T} \mathbf{H}^{-1} \mathbf{L}_{m}
$$

where $\mathbf{L}_{m}$ is the Choleski factor of $\Sigma_{m}^{-1}$. Given that

$$
\begin{aligned}
\hat{\mathbf{o}}(t) & =\mathbf{o}(t)-\hat{\mu}_{m}=\mathbf{o}(t)-\hat{W} \xi_{m} \\
\theta_{m}^{n u m}\left(\hat{\mathcal{O}}^{2}\right) & =\sum_{q=1}^{Q} \sum_{t=s_{q}}^{t=e_{q}} \gamma_{q m}(t) f\left(\gamma_{q}^{M P E}\right) \hat{\mathbf{o}}^{2}(t) \\
\theta_{m}^{\text {den }}\left(\hat{\mathcal{O}}^{2}\right) & =\sum_{q=1}^{Q} \sum_{t=s_{q}}^{t=e_{q}} \gamma_{q m}(t) f\left(-\gamma_{q}^{M P E}\right) \hat{\mathbf{o}}^{2}(t)
\end{aligned}
$$

the auxiliary function in Eq. (3) could be rewritten as below:

$$
\begin{aligned}
& \mathcal{G}(\mathbf{H}, \hat{\mathbf{H}})= \\
& \sum_{r}^{R} \sum_{q=1}^{Q} \sum_{t=s_{q}}^{t=e_{q}} \gamma_{q m}(t) f\left(\gamma_{q}^{M P E}\right) \log \mathcal{N}\left(\mathbf{o}(t), \hat{\mu}_{m}, \hat{\mathbf{H}}^{T} \Sigma_{m} \hat{\mathbf{H}}\right) \\
& -\sum_{r}^{R} \sum_{q=1}^{Q} \sum_{t=s_{q}}^{t=e_{q}} \gamma_{q m}(t) f\left(-\gamma_{q}^{M P E}\right) \log \mathcal{N}\left(\mathbf{o}(t), \hat{\mu}_{m}, \hat{\mathbf{H}}^{T} \Sigma_{m} \hat{\mathbf{H}}\right) \\
& +\sum_{m} D_{m}\left[-\frac{1}{2}\left(\log |\hat{\mathbf{H}}|^{2}+\tilde{\Sigma}_{m} \mathbf{L}_{m}^{T} \hat{\mathbf{H}}^{-1} \mathbf{L}_{m}\right.\right. \\
& \left.\left.+\left(\tilde{\mu}_{m}-\hat{\mu}_{m}\right)^{T} \mathbf{L}_{m}^{T} \hat{\mathbf{H}}^{-1} \mathbf{L}_{m}\left(\tilde{\mu}_{m}-\hat{\mu}_{m}\right)\right)\right]
\end{aligned}
$$

Solving the partial differential with respect to $\hat{\mathbf{H}}$, we can obtain the MPE-based diagonal variance transform for each element $\hat{\mathbf{h}}_{(i)}$ :

$$
\begin{aligned}
\hat{\mathbf{h}}_{(i)} & =\frac{\sum_{m}\left\{\frac{1}{\sigma_{m(i)}^{2}}\left[\theta_{m}^{n u m}\left(\hat{\mathcal{O}}_{(i)}^{2}\right)-\theta_{m}^{d e n}\left(\hat{\mathcal{O}}_{(i)}^{2}\right)+D_{m} Z_{m(i)}\right]\right\}}{\sum_{m} \gamma_{m}^{n u m}-\gamma_{m}^{d e n}+D_{m}} \\
Z_{m(i)} & =\tilde{\sigma}_{m(i)}^{2}+\left(\tilde{\mu}_{m(i)}-\hat{\mu}_{m(i)}\right)^{2}
\end{aligned}
$$

where $D_{m}$ has the same definition as used in Eq. (3), $\tilde{\mu}_{m}$ and $\tilde{\sigma}_{m}^{2}$ are transformed mean and diagonal variance by the initial MLLR.

\subsection{The smoothing technique for MPE-based DLT}

The I-smoothing technique was introduced [7] to prevent MPE training from over-training and improve its generalization. The basic idea behind I-smoothing is to incorporate the information from ML statistics as a "prior", so as to smooth the discriminative statistics over each Gaussian component. In our implementation, an extra term $\log P(\hat{W})$ is appended to the auxiliary function in Eq. (3), which is given by ignoring the terms independent of $\hat{W}$ :

$$
\begin{aligned}
& \log P(\hat{W})=\sum_{m}\left[-\frac{1}{2}\left(\tau \log \left|\Sigma_{m}\right|\right.\right. \\
& \left.\left.+\frac{\tau}{\gamma_{m}^{m l}} \sum_{t} \gamma_{m}^{m l}(t)\left(\mathbf{o}(t)-\hat{W} \xi_{m}\right)^{T} \Sigma_{m}^{-1}\left(\mathbf{o}(t)-\hat{W} \xi_{m}\right)\right)\right]
\end{aligned}
$$

where $\tau$ points of statistics are coming from ML training. Hence, the numerator statistics to estimate MPE-based DLT will be modified with the occupancy count $\tau$ :

$$
\begin{aligned}
\gamma_{m}^{n u m^{\prime}} & =\gamma_{m}^{n u m}+\tau \\
\theta_{m}^{n u m}(\mathcal{O})^{\prime} & =\theta_{m}^{n u m}(\mathcal{O})+\frac{\tau}{\gamma_{m}^{m l}} \theta_{m}^{m l}(\mathcal{O}), \\
\theta_{m}^{n u m}\left(\mathcal{O}^{2}\right)^{\prime} & =\theta_{m}^{n u m}\left(\mathcal{O}^{2}\right)+\frac{\tau}{\gamma_{m}^{m l}} \theta_{m}^{m l}\left(\mathcal{O}^{2}\right) .
\end{aligned}
$$

\section{EXPERIMENTS}

\subsection{Supervised adaptation on WSJ}

In our experiments on WSJ, the acoustic models were constructed with ML training on the SI-284 WSJ0+1 training set. The front end used MF-PLP analysis to get the 39-dimensional features, including static cepstra with 1st and 2nd order derivatives. Thus the gender independent cross-word triphone HMMs consist of 6399 tied-states with 12 Gaussians per state. The testing adaptation is performed on 1994 NAB Spoke 3 (s3-dev and s3-eval) task with an enrollment set (40 utterances) and a testing set (about 20 utterances) for each speaker.

The lattice-based framework as used in MPE training is also employed here to estimate MPE-based DLT on the enrollment set. Initially, word lattices are generated by fast decoding on the adapted models (using MLLR after 3 iterations), with a $20 \mathrm{~K}$ WSJ bigram language model (LM). Then the denominator and numerator phonelevel lattices are created by aligning the recognized word lattices and correct transcriptions separately with a unigram LM. The appropriate statistics for MPE-based DLT are accumulated via a forwardbackward pass through the lattices marked with the phone starting/ending time. Thus using the regression-class tree with 16 baseclasses for speech and 1 baseclass for silence, mean and diagonal variance transforms are estimated under both MLLR and MPEbased DLT schemes.

To evaluate MPE-based DLT for supervised adaptation, the full decoding with $5 \mathrm{~K}$ word vocabulary and a bigram LM is operated and then the generated lattices are expanded with a trigram LM for further rescoring. We list the lattice rescoring results after adaptation in Table 1, where H-cri DLT refers to the use of $\mathrm{H}$-criterion for DLT estimation [3]. And M2 means that the word lattices for DLT estimation are re-generated by decoding on the adapted models (using DLT after 3 iterations).

\begin{tabular}{l||c|c|c|c}
\hline \hline Test sets & iterations & MLLR & H-cri DLT & MPE-DLT \\
\hline s3-dev & 1 ite & 13.2 & 12.4 & 12.2 \\
s3-eval & 1 ite & 11.1 & 10.3 & 10.1 \\
\hline s3-dev & 3 ite & 12.4 & 11.9 & 11.8 \\
s3-eval & 3 ite & 10.4 & 10.6 & 10.1 \\
\hline s3-dev & M2 & - & 11.9 & 11.8 \\
s3-eval & M2 & - & 10.1 & 10.0 \\
\hline \hline
\end{tabular}

Table 1. The WER(\%) on NAB Spoke 3 after MLLR, H-criterion DLT and MPE-based DLT adaptation.

It is observed that MPE-based DLT can improve the supervised adaptation by reducing WER absolute $1.0 \%$, in comparison with standard MLLR after 1 iteration. After multiple iterations, MPE-based DLT still outperforms MLLR by decreasing WER absolute $0.6 \%$ for $\mathrm{s} 3-\mathrm{dev}$ and $0.3 \%$ for $\mathrm{s} 3$-eval. It is worth noting 
that the convergence can be improved when comparing MLLR and DLT after 3 ite. vs. 1 ite.. Moreover, MPE-based DLT performs better than H-criterion DLT, since the smoothing term in the auxiliary function ensures its convergence.

\subsection{Unsupervised adaptation on CTS transcription}

The acoustic models were built with MPE training on CTS transcription (76 hours training set). Trained with a HLDA front-end, the speaker-independent triphone HMMs contain 5920 tied-states with 12 Gaussian components per state. For testing, half of the official development set for the 2001 NIST evaluation dev01sub are used with approximate 3 hours of speech. And lattice rescoring rather than full decoding is operated [10] to evaluate the linear transforms estimated under different criteria.

The 1-best Viterbi output after lattice-MLLR adaptation (5 mean transforms and global full variance transform) and confusion network $(\mathrm{CN})$ decoding is then as the hypothesis to generate the reference phone marked lattices for DLT estimation. The denominator word lattices are created with the bigram LM, which are then aligned to phone level lattices. Therefore, 2 mean and diagonal variance transforms are estimated under MLLR scheme, MMI-based DLT (the estimation formulations are identical to that in [4]) and MPE-based DLT scheme individually. The cheating results, where the true transcriptions are used to generate phone level reference lattices for DLT estimation, are also summarized in the following table.

\begin{tabular}{l||c|c|c}
\hline \hline \multicolumn{1}{c||}{ Adaptation } & \multicolumn{2}{c|}{ hypothesis } & true trans \\
\hline & & $+\mathrm{CN}(27.0)$ & \\
\hline \hline MLLR & 27.7 & 27.0 & 26.1 \\
MMI-DLT & 27.5 & 26.8 & 24.8 \\
MPE-DLT & 27.3 & 26.9 & 23.2 \\
\hline \hline
\end{tabular}

Table 2. The WER(\%) on dev01sub for MPE system, after MLLR, MMI-based DLT and MPE-based DLT adaptation.

It can be seen that MPE-based DLT yields absolute $0.4 \%$ WER gain over MLLR, and $0.2 \%$ gain over MMI-based DLT. After CN decoding, MPE-based DLT gives $0.1 \%$ decrease in WER compared with that of the hypothesis. Although MMI-based DLT yields more $0.1 \%$ gain over MPE-based DLT after CN decoding, we believe that MPE-based DLT could perform better than MLLR and MMI-based DLT which is proven from the cheating column. This aspect implies that MPE-based DLT can be developed for unsupervised adaptation with further work.

\section{DISCUSSIONS AND CONCLUSIONS}

This paper has investigated using the MPE criterion for DLT estimation, which can be applied to both supervised and unsupervised adaptations. With the presented weak-sense auxiliary function, the estimation formulations for MPE-based DLT have been derived, where I-smoothing is used to prevent over-training. The experimental results on WSJ task have shown that MPE-based DLT can considerably improve the supervised adaptation performance.

Our ongoing investigations focus on using MPE-based DLT in the unsupervised style. To effectively use the hypothesis for MPEbased DLT estimation, the confidence scores from $\mathrm{CN}$ outputs can be used to accumulate the statistics with high confidence. Alternatively, with the numerator lattices, the most likely lattices can be weighted by posterior probabilities to estimate DLT.

\section{REFERENCES}

[1] C.J. Leggetter \& P.C Woodland (1995). "Maximum Likelihood Linear Regression for Speaker Adaptation of Continuous Density Hidden Markov Models" Computer Speech \& Language, Vol. 9, pp. 171-185.

[2] M.J.F. Gales (1998) "Maximum Likelihood Linear Transformations for HMM-based speech recognition," Computer Speech and Language, vol. 12, pp. 75-98.

[3] L.F. Uebel \& P.C. Woodland (2001). "Discriminative Linear Transforms for Speaker Adaptation," ISCA ITRW on Adaptation Methods for Automatic Speech Recognition, SophiaAntipolis.

[4] A. Gunawardana \& W. Byrne (2001). "Discriminative Speaker Adaptation with Conditional Maximum Likelihood Linear Regression ," in Proc. Eurospeech'01, Scandinavia.

[5] P.C. Woodland \& D. Povey (2002), "Large Scale Discriminative Training of Hidden Markov Models for Speech Recognition," Computer Speech and Language, vol. 16, no. 1, pp. 25-47.

[6] D. Povey \& P.C. Woodland (2002). "Minimum Phone Error and I-Smoothing for Improved Discriminative Training," Proc. ICASSP'02, Orlando.

[7] D. Povey (2003). "Discriminative Training for Large Vocabulary Speech Recognition,” Ph. D. Dissertation (draft), Department of Engineering, University of Cambridge, U.K.

[8] D. Povey, M.J.F. Gales, D.Y. Kim, \& P.C. Woodland (2003). "MMI-MAP and MPE-MAP for Acoustic Model Adaptation," Proc. EUROSPEECH'03, Geneva.

[9] X.D. He \& W. Chou (2003), "Minimum Classfication Error (MCE) Model Adaptation of Continuous Density HMMs, ” Proc. EUROSPEECH 2003, Geneva.

[10] L. Wang \& P.C. Woodland (2003), "Discriminative Adaptive Training Using The MPE Criterion," Proc. ASRU 2003, Virgin Island.

[11] J. McDonough, T. Schaaf \& A. Waibel (2002). "On Maximum Mutual Information Speaker-Adaptation Training," Proc. ICASSP'02, Orlando. 